\title{
THE STRUCTURE OF FLIGHT MUSCLE
}

\section{SARCOSOMES IN THE BLOWFLY}

\section{CALLIPHORA ERYTHROCEPHALA (DIPTERA)}

\author{
DAVID S. SMITH, Ph.D.
}

From the Department of Zoology, University of Cambridge, England. Dr. Smith's present address is the Department of Biology, University of Virginia, Charlottesville, Virginia

\begin{abstract}
The electron microscopic structure of sectioned indirect flight muscle fibers of the blowfly Calliphora is described. Particular attention is paid to the organization of the sarcosomes (mitochondria) of this tissue, and this description is accompanied by an account of the appearance of these bodies in negatively stained preparations. In sectioned material, it has been shown that these sarcosomes are similar to other mitochondria in the disposition of the outer and inner limiting membranes, but that the cristae, confluent with the latter, are unusually regular, and form parallel plates, containing circular fenestrations forming cylindrical channels within the matrix. Negatively stained preparations of disrupted sarcosomes reveal that both the outer limiting membrane and the cristae membranes bear large numbers of small particles, similar in appearance to those described by FernándezMorán and others in various mitochondria. In Calliphora, these particles consist of a subspherical "head" and a cylindrical "stalk," and appear to be arranged on the mitochondrial membranes either randomly distributed, or collected into circular or elongated groups. Recent suggestions concerning the nature of these submitochondrial particles are discussed, and an attempt is made to correlate the aspects of organization of Calliphora sarcosomes, revealed by conventional sectioning of the "intact" structures, and by negative staining of sarcosomal derivatives.
\end{abstract}

\section{IN TRODUCTION}

It has been well established that the "sarcosomes" of muscle fibers correspond, both structurally and functionally, to the mitochondria of other types of cell. It is also clear that there is a general correlation between the sarcosome content of a given fiber, and its oxidative metabolic activity level, and that each of these features is unusually well developed in certain insect flight muscles. Before the development of techniques for electron microscopic study of cell structure, mitochondria were generally identified by their response to staining procedures, in living or fixed material.
However, the electron microscope demonstrated that these bodies may be described in terms of a characteristic configuration of membranes: that each mitochondrion consists of an inner and an outer membrane, the latter being periodically invaginated into the "matrix" of the mitochondrion to define a more or less transversely arranged series of partitions or "cristae." From one cell type to another, mitochondria exhibit considerable variation on this basic structural plan, and the sarcosomes of insect flight muscles, whilst retaining the generalized organization 
mentioned above, are not only unusually large, but may also exhibit considerable complexity in the arrangement of the cristae.

The role played by the mitochondria in the metabolism and energy conservation of the cell is well known. Insect sarcosomes, in common with other mitochondria, have been shown to contain the enzymes of the Krebs or citric acid cycle by means of which breakdown products of glucose and other foodstuffs are oxidized and, in addition, they contain the system of "carrier" enzyme molecules along which electrons, derived from the degradation of food molecules, are passed, ultimately to oxygen to form water which, with carbon dioxide, represents the end-product of oxidative catabolism. This carrier system, consisting of one or more flavoproteins and a series of cytochromes $^{1}$ is collectively known as the electron transfer system or the respiratory chain, and with it are associated other enzymes that conserve the oxidation-reduction energy derived from the passage of electrons along the chain of carrier molecules, through the synthesis of adenosinetriphosphate, the "phosphate-bond energy" of which may then be made available for the carrying out of chemical work throughout the cell.

${ }^{1}$ The relationship between substrate-level and terminal oxidation in insect muscles and other tissues has recently been reviewed: see Gilmour (1961) for details and references. As in mammalian sarcosomes and other mitochondria, the sarcosomes of insect flight muscles appear to contain enzymes, probably flavoprotein in nature (FP), which remove hydrogen from various substrates (S) and transfer electrons to the cytochrome chain:

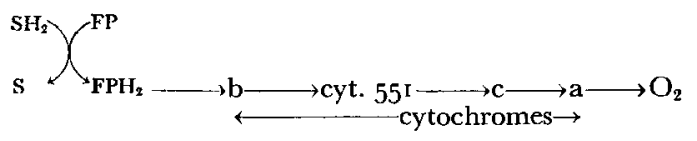

It was formerly thought that in the specialized flight muscles of flies (Chance and Sacktor, 1958) the main terminal respiratory substrate is $\alpha$-glycerophosphate, derived by extramitochondrial glycolysis, the substrate being linked to the cytochrome systern via the flavoprotein $\alpha$-glycerophosphate dehydrogenase. Recently, however, the additional presence of an unusually efficient Krebs cycle pathway has been recognised in these sarcosomes (Van den Bergh, 1962), presumably linked, via the appropriate flavoproteins, to the respiratory chain system.
It appears, furthermore, that while the Krebs cycle enzymes are situated in the mitochondrial matrix, or at least are not tightly bound to mitochondrial membranes, the molecules of the respiratory chain and associated phosphorylating systems are intimately organized into replicated units or assemblies which appear to represent a substantial portion of the organized structural elements or membranes of the mitochondrion. This latter subject has recently been discussed by Lehninger and coworkers (1958), Green (1959), Green and Oda (1961), Lehninger (1962), and others.

During the course of the work described here, it was found that the sarcosomes of asynchronous (fibrillar) flight muscle fibers of the blowfly Calliphora erythrocethala are not only separable with great ease from other components of the fiber, but also show, in the electron microscope, a uniquely regular crista arrangement. Recently, the application of negative staining techniques to a study of mitochondrial fragments (FernándezMorán, $1962 a, b, c)$ has revealed a small particulate component, apparently associated with the mitochondrial membranes, and it has been found that such preparations are readily obtained with Calliphora sarcosomes. Although the present account is concerned primarily with the cytological organization of these sarcosomes, the features mentioned above suggest that this material may prove of value in future studies on the structure and function of the mitochondrial membranes.

\section{MATERIALS AND METHODS}

\section{A. Sectioned Material}

Non-teneral adults of the blowfly Calliphora erythrocephala were employed. Flies were bisected medially and half of the thorax was fixed for two hours in ice cold 1 per cent osmium tetroxide, at $\mathrm{pH} 7.3$ maintained with phosphate buffer (Millonig, 1961 a). The material was transferred to 70 per cent ethanol, at which point fibers of the dorsal longitudinal muscle of the mesothorax were removed, dehydrated, and embedded in Araldite. Blocks were sectioned with glass knives on a Huxley microtome, and sections were stained in lead-tartrate complex solution (Millonig, 1961 b) and examined in a Philips EM 200.

\section{B. Negatively Stained Material}

Flight muscle fibers of Calliphora, in common with asynchronous muscles of members of other orders, contain only a very limited amount of non-mito- 
chondrial membranous material in the sarcoplasm. For preparation of the very small amounts of material required for electron microscopic examination of sarcosome fragments, the usual differential centrifugation procedures for the isolation of mitochondria were dispensed with. The dorsal longitudinal flight muscle was dissected from a chilled fly and was placed on a microscope slide in a drop of (i) deionized water or (ii) $0.18 \mathrm{M}$ phosphate buffer increased to 0.33 $\mathrm{M}$ by addition of sucrose. A second slide was then placed on top of the preparation, and the muscle was crushed for a few seconds; the slides were separated and the macerated tissue was agitated in an additional drop of the isolation medium. When the fibrillar debris was removed with forceps, a turbid suspension consisting of a virtually pure preparation of sarcosomes remained. The preparation (on each slide) was then further diluted by addition of one drop of the isolation medium and two drops of potassium phosphotungstate solution ( 1 per cent phosphotungstic acid titrated to $\mathrm{pH} 6.8$ with $1 \mathrm{~N}$ potassium hydroxide (Horne, 1961; Horne and Whittaker, 1962). Ice cold solutions were employed throughout. The sarcosome suspension was agitated with a glass needle, and placed in a refrigerator for 1 to 5 minutes, after which time drops were removed with a micropipette and placed on collodion-carbon coated grids, allowed to stand for one minute, then withdrawn. For preparation of fixed material, a drop of 1 per cent osmium tetroxide was added to the sarcosome suspension before addition of the phosphotungstate. This procedure results in an even spreading of sarcosomes or their derivatives over the supporting film, and in most places the droplets of phosphotungstate in which the sarcosomes are embedded are sufficiently thin to permit examination of structural details of negatively stained sarcosome components. These preparations are free from myofibrillar contamination, although tracheoles are occasionally present.

\section{OBSERVATIONS}

\section{A. Sectioned Material}

The fibers of the dorsal longitudinal flight muscle of Calliphora have a roughly rectangular cross-section, the dimensions of which are ca. 200 to $300 \mu \times 500$ to $750 \mu$. The surface of each of these very large fibers is deeply grooved or indented (Tiegs, 1955) to accommodate extracellular tracheal branches. The cylindrical fibrils are $c a .2 \mu$ in diameter and, as described by Huxley and Hanson (1960), their constituent myofilaments form a double hexagonal array, differing from that found in vertebrate muscle in that the secondary (actin) filaments lie opposite to and midway between the larger primary (myosin) filaments. As in other asynchronous insect flight muscles, the I bands in Calliphora are very narrow, accounting for less than 10 per cent of the presumed "relaxed" sarcomere length of $3.0 \mu$ (Fig. 1). Much of the interfibrillar sarcoplasm in these fibers is occupied by the sarcosomes. In other insect flight muscles (Smith, $1961 b$; 1962) the sarcosomes may be oriented singly, or in twos or threes, opposite each sarcomere, but in Calliphora, as in Tenebrio (Smith, $1961 \mathrm{a}$ ), these are not precisely aligned with respect to the myofibrillar striations (Fig. 1).

In common with the asynchronous flight muscle fibers of members of other orders (Smith, 1962), the fibers of Calliphora contain a highly modified sarcoplasmic reticulum (SR). The extensive system of tubules and cisternae of the SR present in insect and vertebrate synchronous fibers (Porter and Palade, 1957; Fawcett and Revel, 1961; Revel, 1962; Smith, 1962) is extremely reduced, and appears to be represented by small isolated vesicles (cf. Tenebrio; Smith, 1961 a). As in Tenebrio, an internalized tubular plasma membrane system is present within the fibers of Calliphora flight muscle, derived from the circumtracheolar sheaths drawn into the fiber around the invaginated tracheoles, and these tubules are associated with the isolated vesicles of the SR in two-membered "dyad" structures (Fig. 3) as in Tenebrio. In synchronous insect flight muscle (e.g. Aeshna: Smith 1961 b) on the other hand, the invaginated plasma membrane system probably corresponding to the $T$ system or intermediary component of vertebrate fibers (Andersson-Cedergren, 1959; Porter, 1961) is associated with a well developed cisternal SR, as in vertebrate muscles.

In longitudinal sections of Calliphora flight muscle (Figs. 1 and 2) the sarcosomes are seen as ovoid or irregular profiles, up to $3 \mu$ in length, lying alongside the myofibrils, while transverse sections of the fiber (Fig. 3) indicate that the sarcosomes are tightly insinuated between, and conform to the contours of, the fibrils.

It is now generally agreed that each mitochondrion contains two distinct phases, the matrix and the intracrista spaces, delimited by the membranes of the cristae which are continuous, at least at intervals, with the inner limiting membrane of the mitochondrion. In the case of the sarcosomes shown in Figs. 3 and 7, no transversely sectioned cristae are present: instead, the 
electron-opaque crista surfaces are displayed. These are variegated with light circular areas, of variable size, but with an average diameter of $c a .600 \mathrm{~A}$. The conclusion that such areas represent the surface aspect of perforations or fenestrations in the crista is borne out by sections in which the cristae are displayed in transverse profile. In the latter instance (Figs. 4 and 5) the paired mem brane profiles of each crista are periodically interrupted as they pass, for a short distance, out of the plane of section, at the level of the fenestrations. Not only are the cristae unusually highly oriented (Fig. 4) forming subparallel arrays of about 30 cristae per micron, but, in addition, the fenestrations in successive cristae are precisely aligned one behind the next, as is indicated by sections transverse to the crista face (Fig. 4). This arrangement is illustrated in a diagrammatic reconstruction shown in Fig. 16. Thus, much of the sarcosome matrix in this instance is divided into a series of parallel cylindrical channels, perhaps providing diffusion pathways for transport of metabolites between the lightly-packed cristae. Within a single sarcosome profile, the cristae may be uniformly parallel, though frequently the crista orientation changes abruptly and the cristae included in a single profile pass from transverse to surface aspect, and in the transitional region the channels in the cristae are seen in oblique section (Figs. 1 and 2). The cristae are not precisely oriented with respect to the fibril long axis (Fig. 1) although their arrangement is generally perpendicular or oblique, rather than parallel with this axis; in the transversely sectioned field shown in Fig. 3, for example, several sarcosomes are included, and in each the plane of section is close to that of the crista faces.

Higher magnification electron micrographs (Fig. 5) demonstrate that, as in other mitochondria, the sarcosomes of Calliphora are limited by an outer membrane of simple contour, and an inner membrane defining the outer limits of the cristae. It is evident that each crista, considered as a subcircular plate, is confluent with or invaginated from the inner membrane at intervals around its circumference. This is clearly seen in transversely sectioned peripheral fields (Fig. 5) and especially in the scalloped appearance of the crista margin seen in surface aspect (Fig. 7) (c.f. Fig. 16).

Thus, to summarize, it is clear that in Calliphora flight muscle sarcosomes, as in other mitochondria, the internal volume is divided into two portions or phases: the matrix, lying between the cristae, and the intracrista phase confluent with the narrow peripherally located space between the inner and outer limiting membranes of the mitochondrion. However, in this instance the arrangement of membranes imposes great regularity on the distribution of these two phases. In osmium tetroxide-fixed, Araldite-embedded material, the outer membrane is $c a$. $50 \mathrm{~A}$ in thickness, and the inner membrane, continuous with the cristae, is $c a .70 \mathrm{~A}$ in thickness. In these preparations the triple-layered unit membrane structure has not been detected. If it is assumed that the morphogical partition between the matrix and the interior of the cristae is indeed the osmiophilic membrane described above, and that the extent of the mitochondrial phases has not been greatly altered during specimen prepa-

FIGURe 1 A longitudinal section of dorsal longitudinal flight muscle of the blowfly, Calliphora erythrocephala, illustrating the close juxtaposition of the sarcosomes or mitochondria $(s)$ and a fibril $(f)$. The striations of the latter are indicated $(Z, A, M)$. Note that the sarcosomes are not aligned with the striations. A transversely sectioned tracheole is inserted between two sarcosomes at $t \times 37,000$.

Figure 2 A field similar to that shown in Fig. 1, at higher magnification. A portion of a longitudinally sectioned fibril is included at $f$. The cristae of the sarcosomes of Calliphora flight muscle are subparallel, and take the form of fenestrated plates. The fenestrations of successive cristae are aligned ( $c f$. Fig. 16) and form cylindrical channels within the sarcosome matrix. At 1 the cristae are seen in transverse section, and the channels defined by the fenestrations are longitudinally sectioned. Within this sarcosome profile the orientation of the cristae changes: at 3 the fenestrations are seen in surface aspect, while at 2 the plane of section is oblique with respect to the channels. $\times 58,000$. 

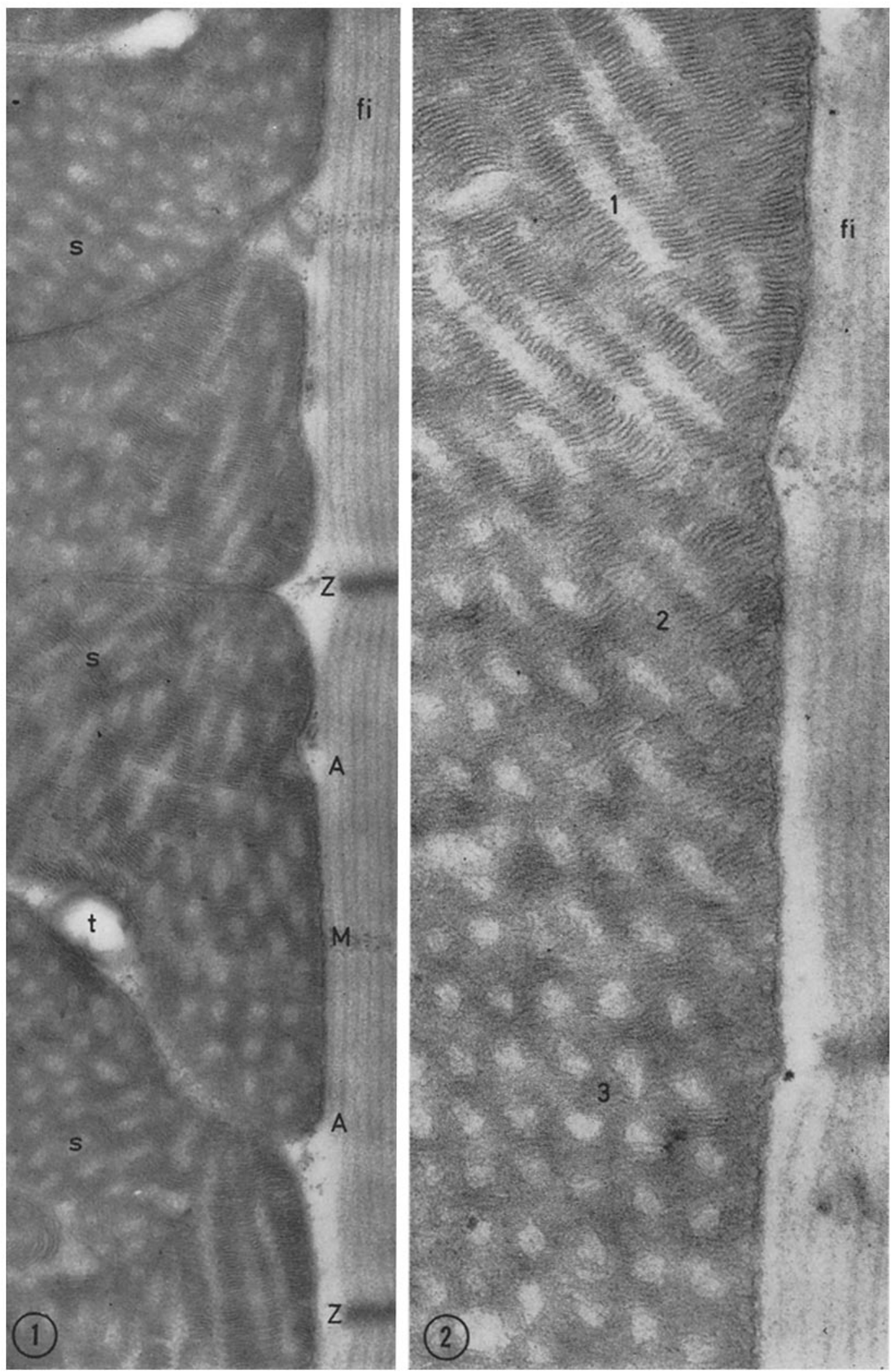

David S. Sмitu Flight Muscle Sarcosomes 


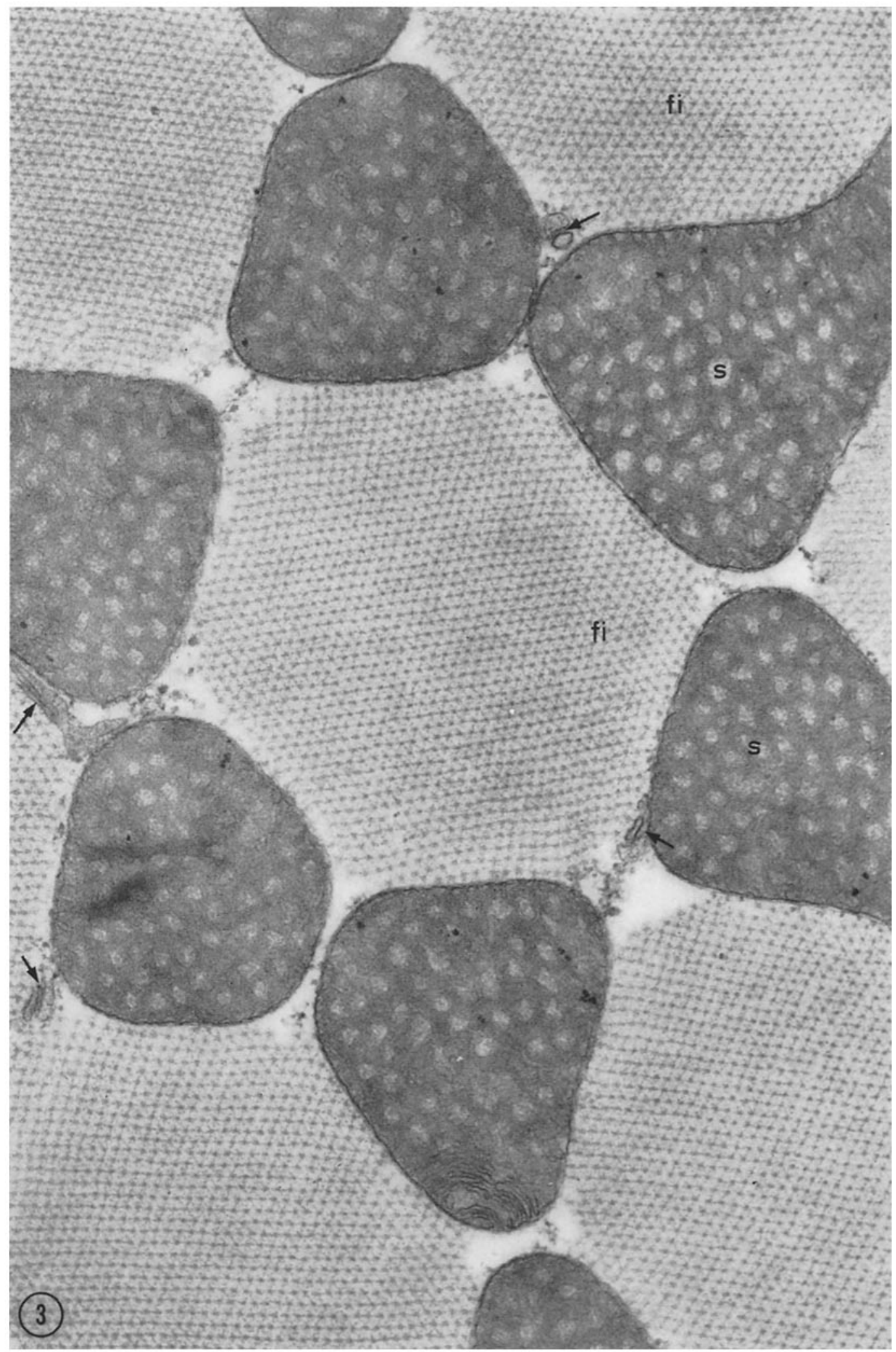

120 The Journal of Cell Biology - Volume 19, 1963 
ration, then the width of the intracrista spaces and of the intercrista matrix is in each case $c a$. 70 to $100 \mathrm{~A}$ (Fig. 4). In sarcosomes of vertebrate skeletal muscles (Andersson-Cedergren, 1959, and others), and indeed in mitochondria in general, the matrix is usually more extensive; that is, the cristae are more widely spaced. The extreme close-packing of the cristae observed in Calliphora, however, is approached by the sarcosomes of other insect muscles (e.g. Aeshna: Smith $1961 b$; Tenebrio: Smith, $1961 a$; Pieris and Polistes: Smith, 1962), and this appears to be a feature of flight muscle fibers, whether synchronous or asynchronous in their stimulationcontraction characteristics. ${ }^{2}$

\section{B. Isolated and Negatively Stained Sarcosomes}

The value of negative staining or negative contrast methods for electron microscopy resides in the fact that if small particles of biological material, or cellular fragments, are suspended in a solution of certain heavy metal salts (e.g., potassium phosphotungstate) and pipetted or sprayed onto a supporting film, the preparation, when dried and examined, reveals the particles or other struc-

${ }^{2}$ Green and Hatefi (1961) have drawn attention to the fact that close packing of the cristae within a mitochondrion reflects not only a high oxidative metabolic rate, but also a high degree of functional specialization, that is, reduction of secondary synthetic ATP-dependent functions, e.g. phospholipid and protein synthesis, etc. Green and Hatefi (1961) mention mammalian heart muscle sarcosomes as an example of this type of mitochondrion, and contrast this with the "versatile" mitochondrion of the liver cell which has very sparsely distributed cristae. Insect flight muscle sarcosomes clearly conform well to this structural generalization, and also appear to be biochemically specialized in the efficiency of their phosphorylating systems and in the specificity of their substrate requirements ( $c f$. Van den Bergh, 1962). tures as more or less transparent objects, embedded in sheets or droplets of the electron-opaque salt (Brenner and Horne, 1959; Horne, 1961; Horne and Whittaker, 1962). Furthermore, the heavy metal salt penetrates into and reveals any structural "sculpturing" or differentiation of the stained object, which might otherwise be unresolvable. This method, originally employed in studies of viruses and bacteria (Brenner and Horne, 1959) has recently been applied successfully to particulate fractions of brain homogenates (Horne and Whittaker, 1962) and to mitochondrial fragments (Fernández-Morán, 1962 a, b, c; 1962), and evidently affords an important complement to observations on cell organelles and other biological structures carried out on sectioned material.

If the sarcosomes of Calliphora flight muscle fibers are isolated in the $0.33 \mathrm{~m}$ medium before addition of phosphotungstate solution, most of them appear to be collapsed but unruptured, when examined in the electron microscope (Fig. 8). In material thus prepared it is difficult to determine whether the stain has penetrated the sarcosomes or is situated in declivities on the surface of the collapsed structures. These preparations probably correspond to the negatively stained mitochondria from brain homogenates described by Horne and Whittaker (1962). In the latter instance the organelle is smaller and less complex than in the case of the Calliphora sarcosome, and the subparallel arrangement of the cristae is clearly seen, but it is probable that the subparallel striations seen in Fig. 8 represent the edges of the cristae of this large and complex structure. However, in material isolated and stained in this way, no further mitochondrial structure has been resolved. When, on the other hand, the sarcosomes are isolated in deionized water, the negatively stained preparations have a greatly different appearance; in particular, the majority are found to be rup-

Figure 3 A transverse section of Calliphora flight musele. The fibrils $(f)$ are cylindrical, and profiles of sarcosomes $(s)$ are inserted between them. In this field, the cristae of the sarcosomes are parallel with the plane of section, and the fenestrations are thus seen in surface aspect, as light areas on the face of the cristae, generally arranged in rows. As in other asynchronous insect flight muscle, the sarcoplasmic reticulum in Calliphora fibers is highly modified, compared with that of vertebrate muscle and synchronous insect muscle, and is represented by two-membered "dyad" associations (arrows) as in Tenebrio ( $f$ Smith, 1961 a). Internalized tracheoles are abundant throughout the fiber in Calliphora, but do not occur in the field shown here. $\times 40,000$. 

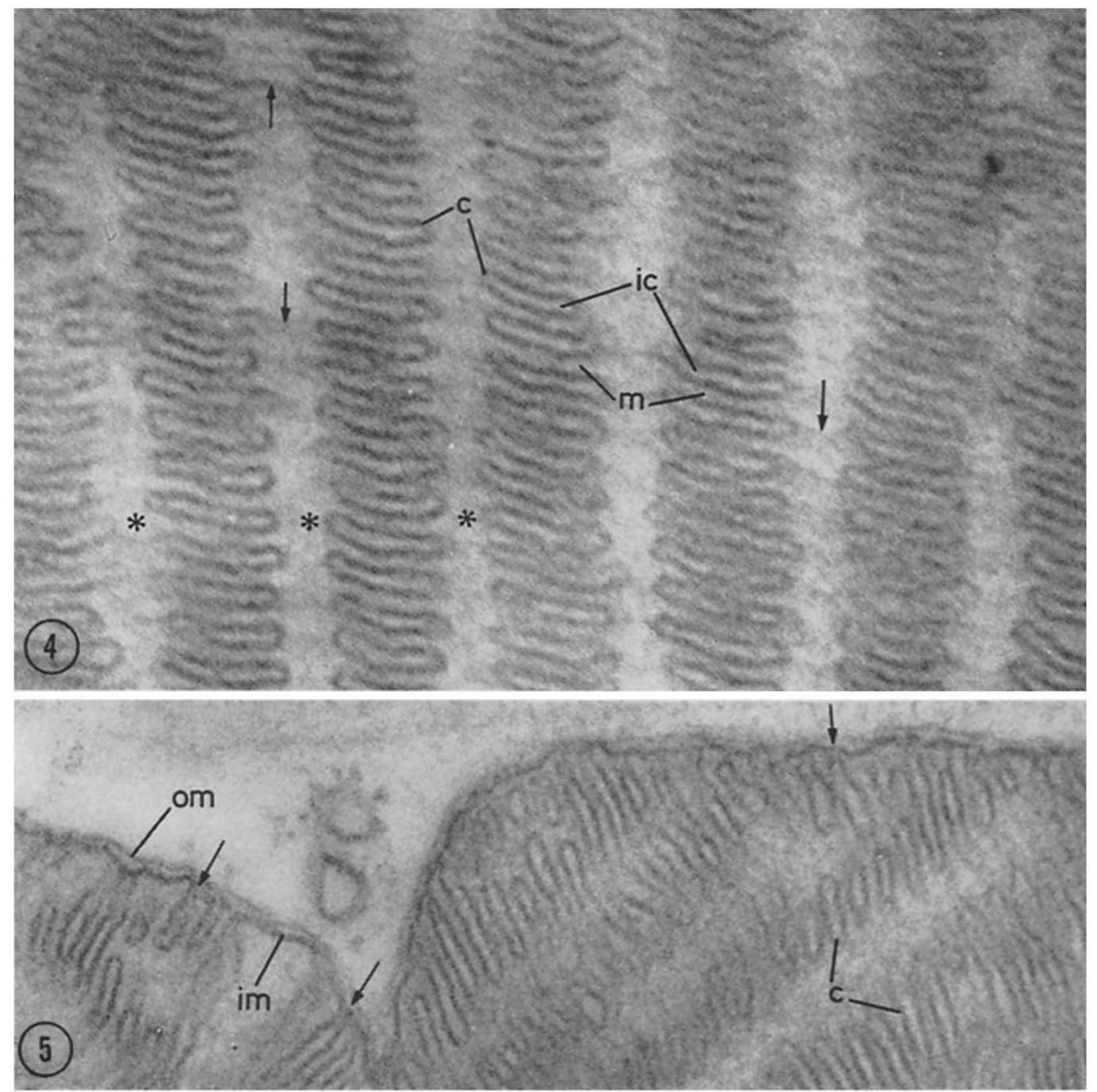

Figure 4 A portion of a sarcosome profile in which the plane of section is perpendicular to the face of the cristae, and which hence includes longitudinal profiles of the channels defined by the crista fenestrations ( $c f$. Fig. 16). The membranes of the cristae (c) are $c a .70 \mathrm{~A}$ in thickness, and the intervening spaces, the matrix of the sarcosome $(m)$ and the intracrista spaces $(i c)$, are each $c a .100 \mathrm{~A}$ wide. This section passes more or less diametrically through several series of crista fenestrations $\left(^{*}\right)$ : along these channels, the membrane edges limiting the fenestrations are faintly visible (arrows) in the depth of the section. The arrangement of cristae in these sarcosomes is unusually regular, but is essentially similar to that in other mitochondria ( $c f$. Fig. 5). $\times 150,000$.

Figure 5 A peripheral region of two Calliphora sarcosomes. As in other mitochondria, both an outer membrane $(\mathrm{om})$ and an inner membrane $(\mathrm{im})$ surround these structures. The latter membrane is confluent with the cristae (c) at intervals, as at the points indicated with arrows. In surface views of the cristae, the periodical links with the inner limiting membrane result in a scalloped appearance at the crista margin (cf. Fig. 7). $\times 115,000$. 
tured or fragmented, and the following account concerns material prepared in this manner.

It should be pointed out here that the identification of the structures described as sarcosome derivatives is well founded. If a gently teased fragment of fresh Calliphora muscle is examined in phase contrast, very large numbers of subspherical bodies are seen flowing away from the myofibrils. These have long been recognised as the mitochondria of the fiber, and represent virtually the sole structural component of the preparations described here. As in striated muscle in general, insect flight muscle cells are almost devoid of ribosome-associated membranes, and, in addition, asynchronous fibers (such as in Calliphora) contain little of the agranular cisternal system of the sarcoplasmic reticulum occurring in vertebrate muscle and synchronous insect muscles. In short, from the evidence obtained in sectioned material, it is clear that the possibility of confusion between mitochondrial and other membranous derivatives can hardly arise, in these preparations of Calliphora flight muscle.

The appearance of Calliphora sarcosomes in deionized water, observed in phase contrast, corresponds precisely to that described by Harman (1950) for similarly treated mitochondria isolated from kidney tubule cells. In each instance, the mitochondria become swollen and bubble-like, containing a characteristic crescentic region of dense material ( $c f$. Kölliker, 1888). Lehninger (1962) has considered various alternative morphological changes that may take place during mitochondrial swelling, and as yet there appears to be insufficient evidence on which to decide this question. However, of the possibilities outlined by Lehninger, the material described here favors that involving inflation of the space between the inner and outer limiting membranes, that is, by the formation of a distended "bleb" at one side of which lie the cristae ( $c f$. Green, 1959). Harman found that mitochondrial swelling in deionized water is not accompanied by loss of soluble materials into the medium, and phase contrast observations on suspensions of Calliphora sarcosomes, drying out on a slide or on the supporting film on a grid, indicate that these swollen structures are ruptured by surface tension stresses occurring during the final stage of evaporation of the water film. Each sarcosome, represented in free suspension by a bubble including a denser crescent, as mentioned above, yields, on drying, a thin membrane component with sharply defined and often crenelated edges, together with an irregular localized deposit, often consisting of very fine strands. These two components are readily recognised in electron micrographs of such preparations, and, it is suggested, represent, respectively, the ruptured outer limiting membrane of the mitochondrion, and the inner membrane together with the more or less disorganized cristae, the separation of these two components occurring during swelling, as has been suggested by Green (1959).

Portions of typical ruptured sarcosomes are shown, at low magnification, in Figs. 9 and 10. Each such structure appears to consist of a membranous sheet applied to the supporting film, and an array of compact sheets and ribbon-like strands. The former component has sharply defined limits, and its edges are disposed in smooth curves or lobes (Fig. 9), beyond which the ribbons may extend. As suggested above, it is believed that these structures may represent the torn outer mitochondrial membrane, and derivatives of the inner membrane and cristae. In some instances (Fig. 10), the ribbons are seen to be confluent with compact plates, thought to represent relatively intact cristae, and in which the fenestrations, described in sectioned material, may be recognised. The technique of negative staining reveals certain structural details of these membranous elements; these will be described first, and later an attempt will be made to relate these findings to observations on the organization of the intact sarcosome.

The membranous ribbons, such as those in the survey micrographs (Figs. 9, 10 and $12 a$ ), are seen at higher magnification in Figs. 13 and 14. Each ribbon includes an axial structure, $c a .100 \mathrm{~A}$ in minimum width but expanded at irregular intervals. Each ribbon is flanked on either side by a series of light subcircular units embedded in the electron-opaque phosphotungstate matrix. These represent the negatively stained images of presumably spherical or slightly ovoid particles, each 80 to $95 \mathrm{~A}$ in diameter. This submitochondrial unit was first described by Fernández-Morán (1962 $a, b, c)$ in similarly stained dried or hydrated mitochondrial preparations.

In the Calliphora preparations, it may be seen that, as in Fernández-Morán's material, each particle is produced in a stalk, 40 to $50 \mathrm{~A}$ in length and 30 to $40 \mathrm{~A}$ in diameter (Fig. 14), 

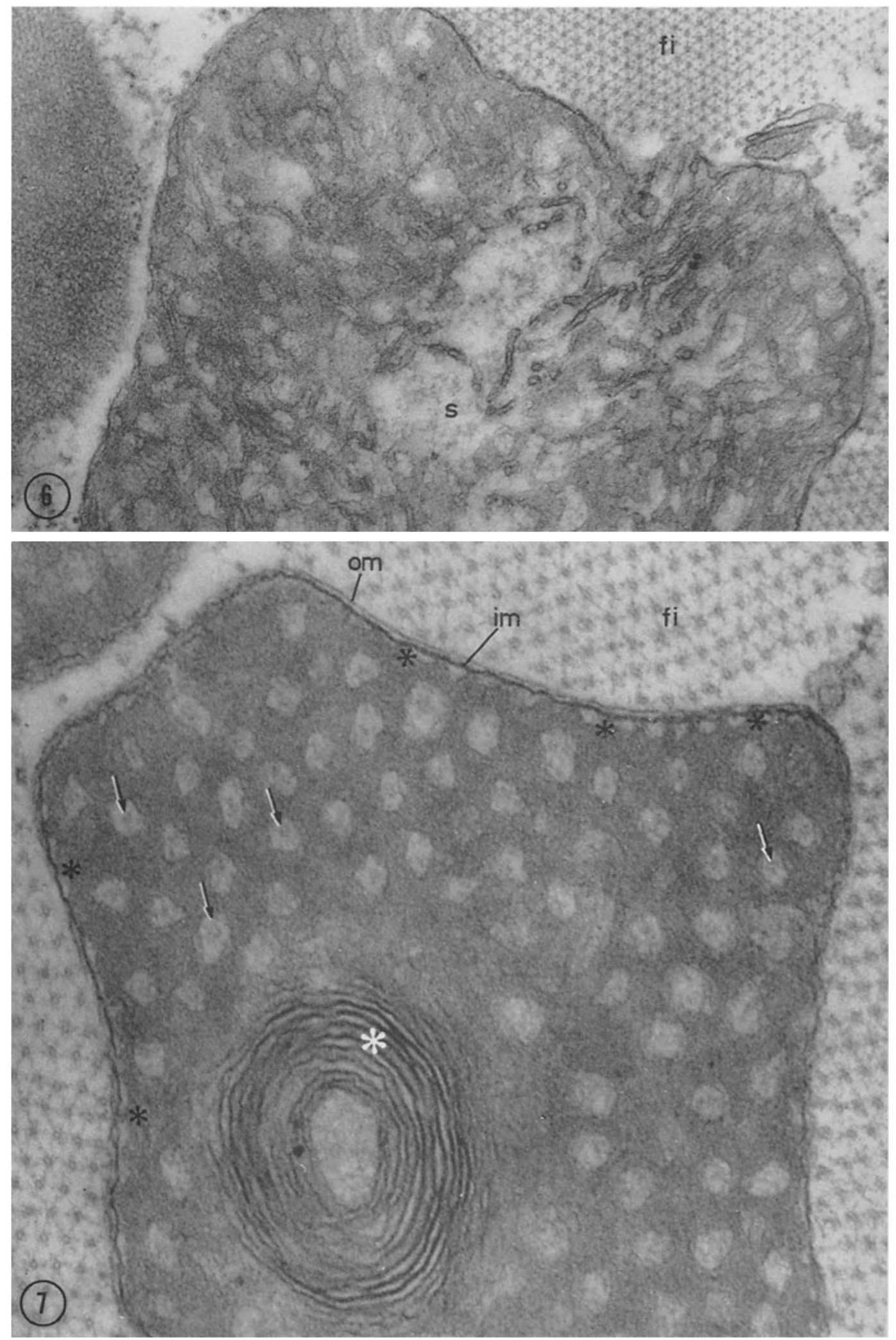

124 The Journal of Cell Biology - Volume 19, 1963 
seemingly linking the particle to the axis of the ribbon. There is also some indication that the axis of the ribbon is differentiated, at the point of attachment of each stalk, into a "dumb-bell" configuration. In regions where the axis is very narrow (Fig. 14) it exhibits a median dense line, but this disappears where the ribbon expands and is presumably unrelated to 'unit-membrane' organization. The broader regions of the ribbons are studded with particles, in addition to those laterally placed and lying directly against the supporting film. ${ }^{3}$

An extensive field of these ribbons is illustrated in Fig. 9, while Figs. 10 and $12 a$ show regions of confluence between these structures and the compact formations identified as cristae. An extension of the area included in Fig. $12 a$ is shown, at higher magnification, in Fig. 12, in which a complex organization is visualized. The latter field contains several irregular densely stained areas ( $c f$. Fig. 10) which may represent fenestrations of the crista, and the remainder of the area of the

3 To supplement the Calliphora preparations described here, sarcosome isolates were obtained from flight muscles of the beetle Tenebrio molitor and the butterfly Nymphalis io. Examination of sectioned fibers indicates that the crista arrangement of these last two species is less regular than in Calliphora, but the appearance of negatively stained sarcosome fragments, after isolation in deionised water, was similar in all three instances. A low magnification field including particle-studded ribbons derived from a Nymphalis sarcosome is shown in Fig. 15. field comprises a membranous substrate, bearing large numbers of particles similar in size and appearance to those occurring alongside the ribbons. Many of these particles are closely associated with circular or elongate figures, $c a .150$ to $300 \mathrm{~A}$ in diameter or up to several hundred Angstroms in length. Each of these figures is surrounded by a halo of stalked particles, and, in addition, other particles are scattered profusely but apparently randomly over the surface of the membranous substrate, that is, it is suggested, over the surface of the crista. At several places in Fig. 12, the particle-associated figures project into the perforations or fenestrations of the substrate, a circumstance that may be related to the presence of indistinct structures observed within the fenestrations of the cristae of sectioned sarcosomes seen in surface aspect (Fig. 7).

Fig. 10 illustrates the topographical relationship between the compact or intact crista membranes and the ribbons, and these components lie above the membranous sheet believed to represent the ruptured outer mitochondrial membrane. A portion of this field is reproduced at higher magnification in Fig. 11. In this instance the two relatively intact cristae are rather heavily stained, but are seen to bear a profuse array of particles and also the circular or elongate figures described above ( $c f$. Fig. 12). The latter are especially clearly seen on the outer membrane, where not only are the figures and particles apparent, but the narrow stalks linking these structures, in the

FIGURE 6 Although the majority of sarcosomes in any section of Calliphora flight muscle show a highly regular structure, disorganized sarcosomes, such as that illustrated here, are occasionally met with. It is thought that such sarcosomes may be degenerating. Traces of the crista fenestrations remain, but, for the most part, the crista membranes are represented by loose aggregates of tubular elements. It is possible that a similar disruption of the cristae takes place when the sarcosome swells, in deionised water, and is ruptured during drying, resulting in the formation of the "ribbons" observed in the negatively stained preparations described and illustrated here ( $c f$. Figs. 9 and 10). $\times 60,000$.

Figure 7 Illustrating a sarcosome profile in which a crista is displayed in surface aspect (cf. Fig. 3). Note the distinct inner and outer limiting membranes ( $\mathrm{im}$, om) ( $c f$. Fig. 5). Each crista is derived from, or confluent with, the inner limiting membrane at intervals around the crista perimeter, resulting in the "scalloped" appearance of the crista margin (black asterisks). The fenestrations of the crista are subcircular, and often contain a poorly defined structure (arrows) ( $c f$. Fig. 12). Regions of the sarcosome containing whorled arrays of membranes such as that seen here (white asterisk) are frequently observed in this material, and these membranes appear to be continuous with those of the cristae. $\times 92,000$. 


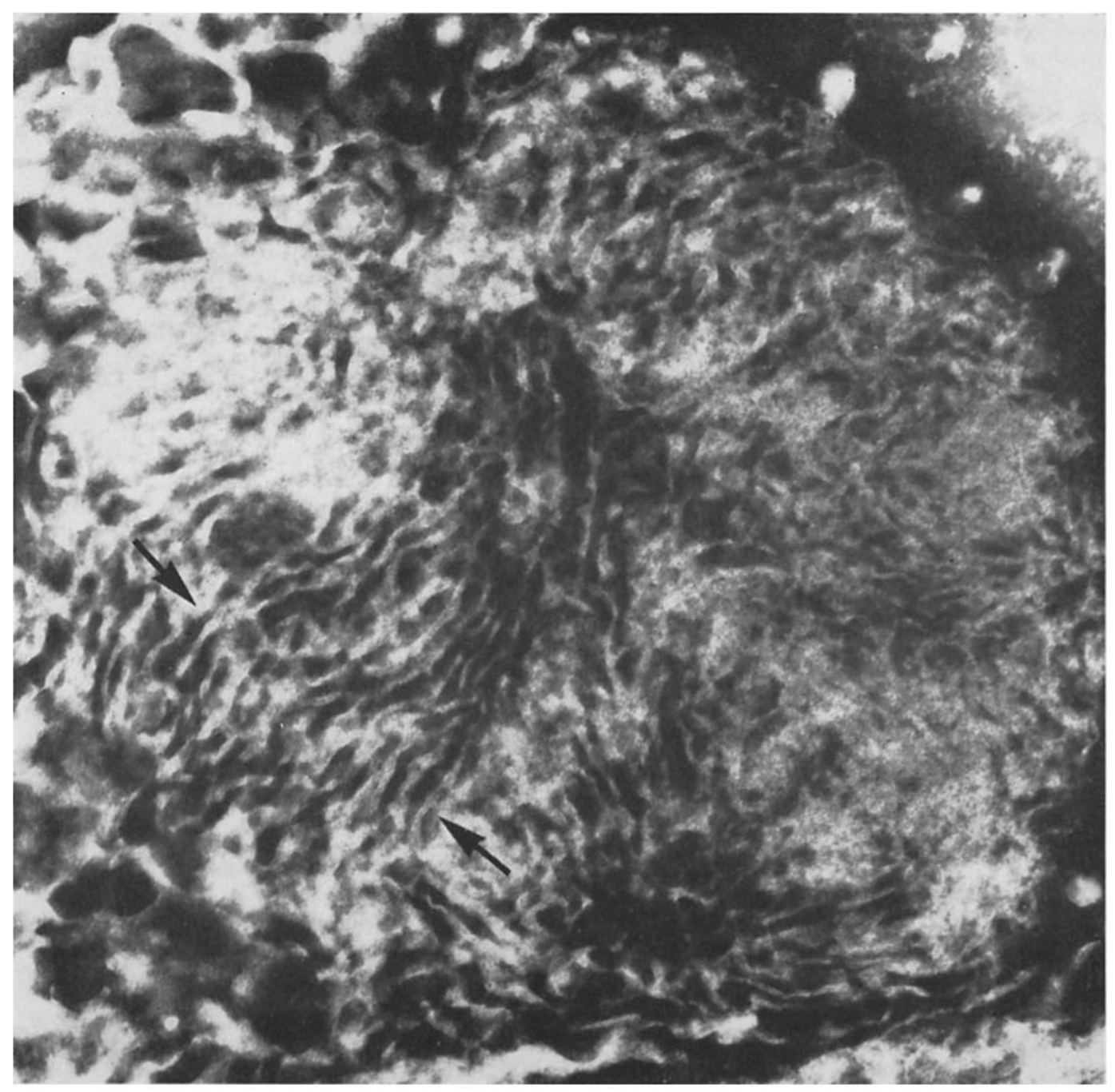

FIGURE 8 An electron micrograph of a sarcosome from Calliphora flight muscle, isolated in $0.33 \mathrm{~m}$ medium and negatively stained with potassium phosphotungstate solution. Under these conditions the majority of the sarcosomes are not disrupted ( $c$. Figs. 9 through 15), and little structural detail may be seen within the collapsed sarcosome. It is difficult to determine whether the stain has penetrated the structure or is solely deposited on the irregular surface of the sarcosome; however, arrays of parallel lines or striations are often seen (arrows), perhaps representing the edges of cristae. $\times 50,000$

FIGURE 9 A low-power electron micrograph illustrating a portion of a ruptured sarcosome from Calliphora flight muscle, negatively stained with phosphotungstate. N.B. This specimen, and those shown in Figs. 10 through 15, represent sarcosome derivatives that were isolated in deionised water, prior to addition of the stain (ef. Fig. 8).

The membranous fragments of the sarcosome lie against the collodion-carbon supporting film $(s f)$. Directly against the latter is present a membranous sheet, believed to represent the ruptured outer mitochondrial membrane $(\mathrm{om})$. Above this is situated a series of ribbonlike structures (white asterisks) interpreted as fragments of the cristae. At lower right, these ribbons ( $r$ ) extend beyond the edge of the outer membrane component (black arrows) onto the supporting film. Higher magnification micrographs of these ribbons are shown in Figs. 13 and 14, but, even at the magnification illustrated here, it may be seen (white arrow) that each of these structures is associated with a series of small particles. $\times 75,000$. 


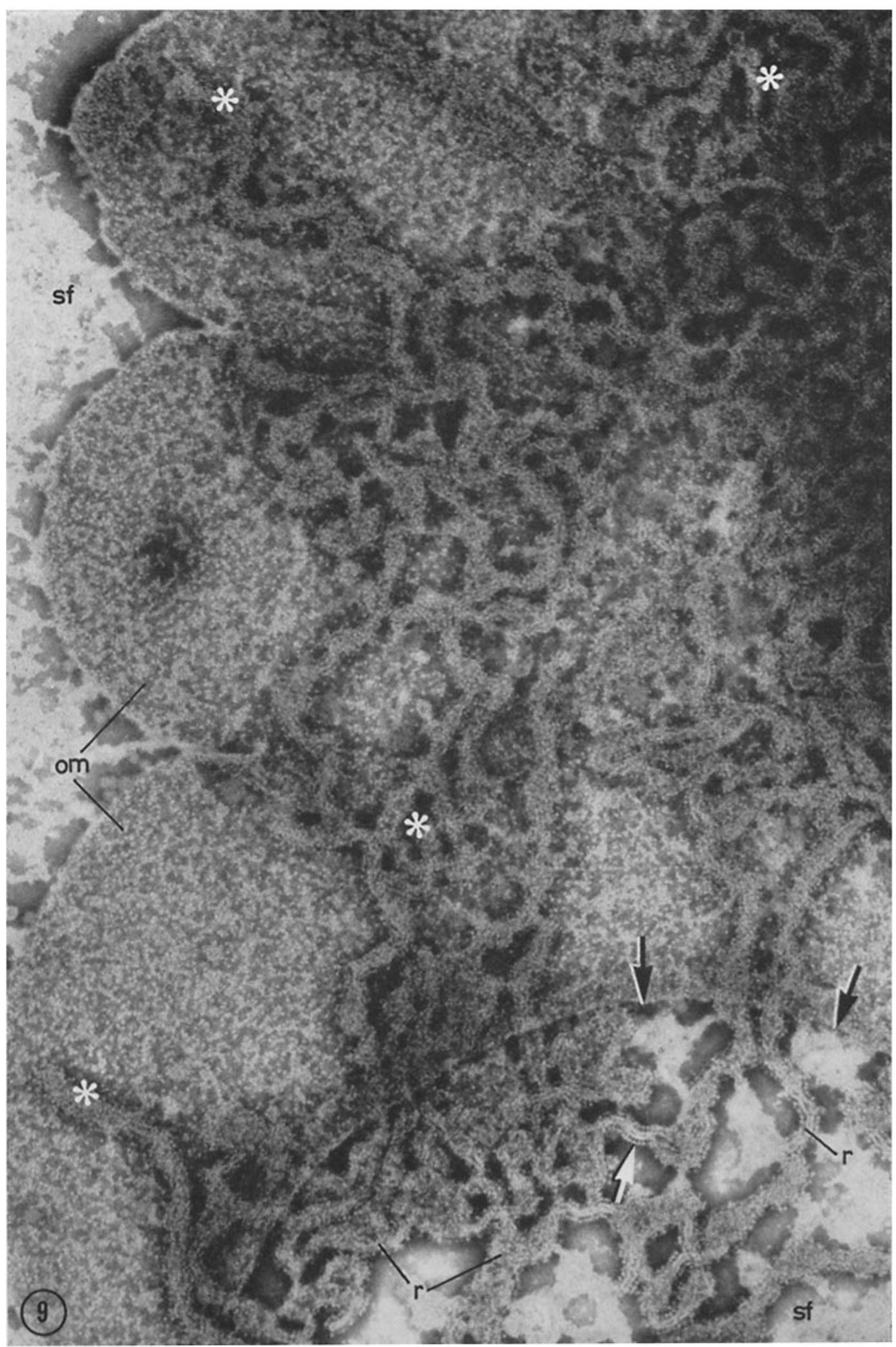

David S. SмIth Flight Musele Sarcosomes 
configuration observed in the ribbons ( $c f$. Figs, 13 and 14), are resolved. The nature of these figures is unknown; indeed, it must be stressed that neither they nor the particles described above have been identified in the sectioned material described here, although Fernández-Morán $(1962, b, c)$ has detected circular or polyhedral components, similar in size to the particles described above, in sections of heart muscle sarcosomes, fixed in osmium tetroxide, and examined at low beam-intensity coupled with specimen coating.

It is possible, on the basis of the identification of these sarcosomes components adopted here, and from measurements made on sectioned material and negatively stained preparations, to estimate the membrane surface area and particle count per unit volume of the sarcosome. The fenestrations occur along the cristae in a concentration of about $80 / \mu^{2}$, and their average diameter is about $0.06 \mu$. The cristae are present in arrays of about $30 / \mu$. Thus, each cubic micron of a sarcosome contains about $60 \mu^{2}$ of crista membrane surface less the area occupied by the fenestrations of the 30 cristae; that is, about $53 \mu^{2}$ of membrane surface per $\mu^{3}$. Counts made on negatively stained preparations of the more intact cristae (excluding the fenestrations) indicate an approximate concentration of particles over the crista surface of $4000 / \mu^{2}$, and a similar figure is obtained in the case of the outer membrane elements. On the basis of these estimates, it has been calculated that each cubic micron of the sarcosome may contain about 200,000 particles. If each stalked particle is considered to be equivalent to a sphere $100 \mathrm{~A}$ in diameter, it may be further calculated that the particles account for about 10 per cent of the total volume of these mitochondria. These sarcosomes are generally somewhat irregular in shape and size, but the volume of each is in the range of $0.5 \mu^{3}$ to several $\mu^{3}$, and thus the number of particles contained within a single sarcosome is of the order of $10^{5}-10^{6}$ (cf. the figure of $10^{4}-10^{5}$ estimated by Fernández-Morán from examination of smaller mitochondria).

Despite the present difficulty in interpreting and interrelating the results afforded by these different methods of study, it may be said that, in the negatively stained preparations described here, all the membrane fragments, whether derived from the outer or inner membrane system, contain a particulate population of uniform size and appearance, associated with or borne on a substrate, either randomly distributed or precisely patterned in conformity with a structurally distinct differentiation of the substrate.

\section{DISCUSSION}

While it is probable that negative staining techniques will become increasingly valuable in studies of the organization of cell fractions, it is necessary, at this stage, to exercise caution in attempting to interpret the preliminary results at present available. These methods may provide information on organization at the macromolecular level, but it must be remembered that such organization may be altered, and artefacts introduced, during isolation and disruption of the cell and the subcellular components.

The presence of a well defined particulate component associated with the membrane fragments of Calliphora sarcosomes, in the preparations described here, is beyond dispute; but the descriptive portion of this work must be clearly distinguished from conjecture on the nature or identity of the structures seen in the phosphotungstate preparations. Fernández-Morán in a preliminary note (1962 a) and in more detailed accounts $(1962, b, c)$, has described units or "elementary particles" associated with the membranes of negatively stained mammalian mitochondria, and he has suggested that these particles may represent the respiratory chain assemblies. It is

Figure 10 A preparation similar to that shown in Fig. 9. Above the sheet representing the outer membrane $(\mathrm{om})$ lie arrays of ribbons, and these are confluent (white arrows) with compact subcircular structures $\left(c_{1}, c_{2}\right)$ interpreted as cristae that have remained partially intact. At upper right (black arrows) the edges of these structures are seen above the outer membrane. The deeply stained circular areas on the surface of the uppermost crista (white asterisks) probably represent the fenestrations seen in sectioned material ( $c f$. Figs. 3, 7 etc.).

The rectangular boxed area in this field is reproduced at higher magnification in Fig. 11. $\times 80,000$. 


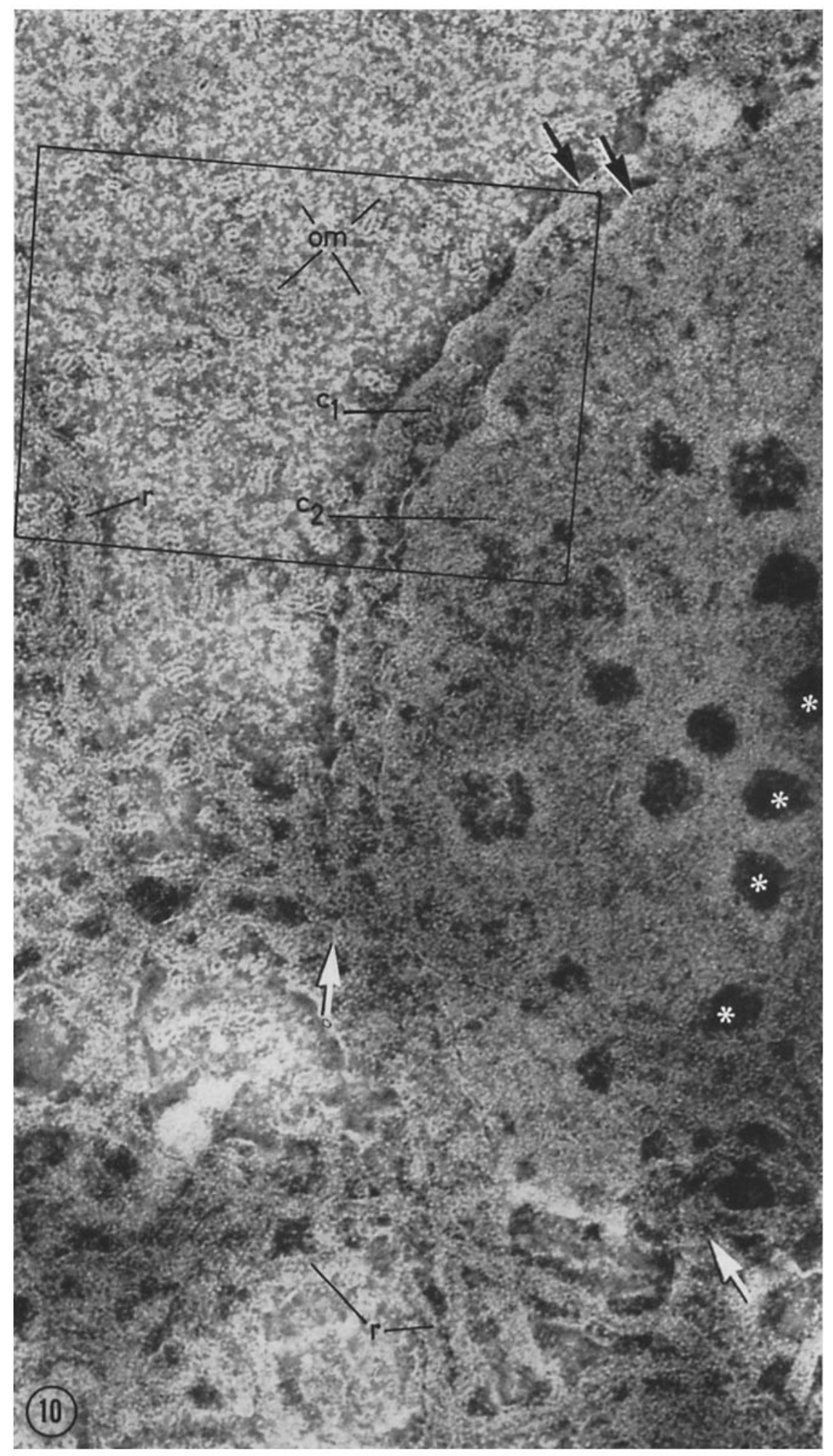

David S. Sмith Flight Muscle Sarcosomes 
clear that the particles from insect and mammalian sources are of similar size. No attempt has yet been made to carry out correlated biochemical and electron microscopic studies on the insect sarcosome preparations, and thus no experimental basis exists at present for the characterization of the particles from these sarcosomes. Furthermore, although suggestive evidence is accumulating (Fernández-Morán, 1963; Green et al. 1963) it does not seem that the nature of particles from mammalian tissue has been rigorously demonstrated. However, as a basis for later studies on the structural organization of the insect sarcosome, a brief comparison between the biochemical properties of insect and vertebrate mitochondria may be apposite.

The enzymes of the citric acid cycle have been shown to be localized in insect sarcosomes, as in other mitochondria, by Watanabe and Williams (1951) and Sacktor (1955) and others, and a detailed account of the recognition of individual components of this cycle has been given by Gilmour (1961). Similarly, the flavoprotein and cytochrome components of the respiratory chain in insect sarcosomes appear to differ only in detail from the corresponding system in other tissues (references in Gilmour, 1961), and the same is true of the phosphorylating systems involved in ATP synthesis.

It is well known that in mitochondria isolated from mammalian tissues the mechanisms of oxidation and phosphorylation, obligatorily linked in the intact organelle, may be uncoupled by mechanical damage to the mitochondrion produced by swelling or other means, suggesting that such damage interferes with the spatial ordering of the enzyme components. Despite the extremely high rate of oxygen consumption of actively flying insects, and notably those with asynchronous flight muscles (e.g. Diptera and Hymenoptera), insect sarcosomes have not, as Gilmour (1961) points out, proved the ideal system for in vitro studies on substrate oxidation and oxidative phosphorylation. However, it appears that insect sarcosomes are unusually fragile and sensitive to isolation procedures, a feature to which the low respiratory level and phosphorylative ability of earlier preparations may, in part at least, be attributed. With improved techniques of study, high levels of oxidative ability, respiratory control, and ATP synthesis have now been obtained in some preparations of insect sarcosomes (references in Gilmour, 1961). It has also been shown (Van den Bergh, 1962) that, in addition to the fragility of insect sarcosomes (in this case from flight muscle fibers of the housefly Musca), these mitochondria may be biochemically very specialized and, when supplied in vitro with the correct substrates, are able to consume oxygen and phosphorylate very efficiently.

Thus, not only is it clear from sectioned material that insect sarcosomes conform to the generalized mitochondrial plan, in terms of the disposition of their membranes, but also it appears that their enzymatic organization is in general similar to that of other mitochondria. Therefore, the results obtained with these various mitochondria by negative staining and other methods may be compared directly, and a common interpretation of the structures revealed by these methods may reasonably be sought.

Whereas the enzymes of the Krebs cycle are situated in the mitochondrial matrix, or are at least readily released from the mitochondrion when the structure is ruptured or fragmented, the respiratory chain carriers and the enzymes effecting phosphorylation are tightly associated with the mitochondrial membranes. It has been suggested (Ogston and Smithies, 1948; Chance and Williams, 1956; Lehninger et al., 1958) that the electron transfer carrier protein molecules are intimately organized into replicated units or

FIgure 11 A portion of the field shown in Fig. 10, at higher magnification. The edges of the two plate-like structures believed to represent cristae are indicated by white asterisks. The expanse of outer membrane, occupying the bulk of this field, bears large numbers of small particles (seen in these preparations as electron-transparent units), both randomly dispersed, and associated into groups surrounding small circular or elongate figures (thick arrows). The cristae are likewise covered with similar particles, either scattered or (thin arrows) in groups. At upper left is included a portion of a membranous ribbon, derived from a disrupted crista, and this structure is also particle-studded (cf. Figs. 13 and 14) (double arrows) $\times 200,000$. 


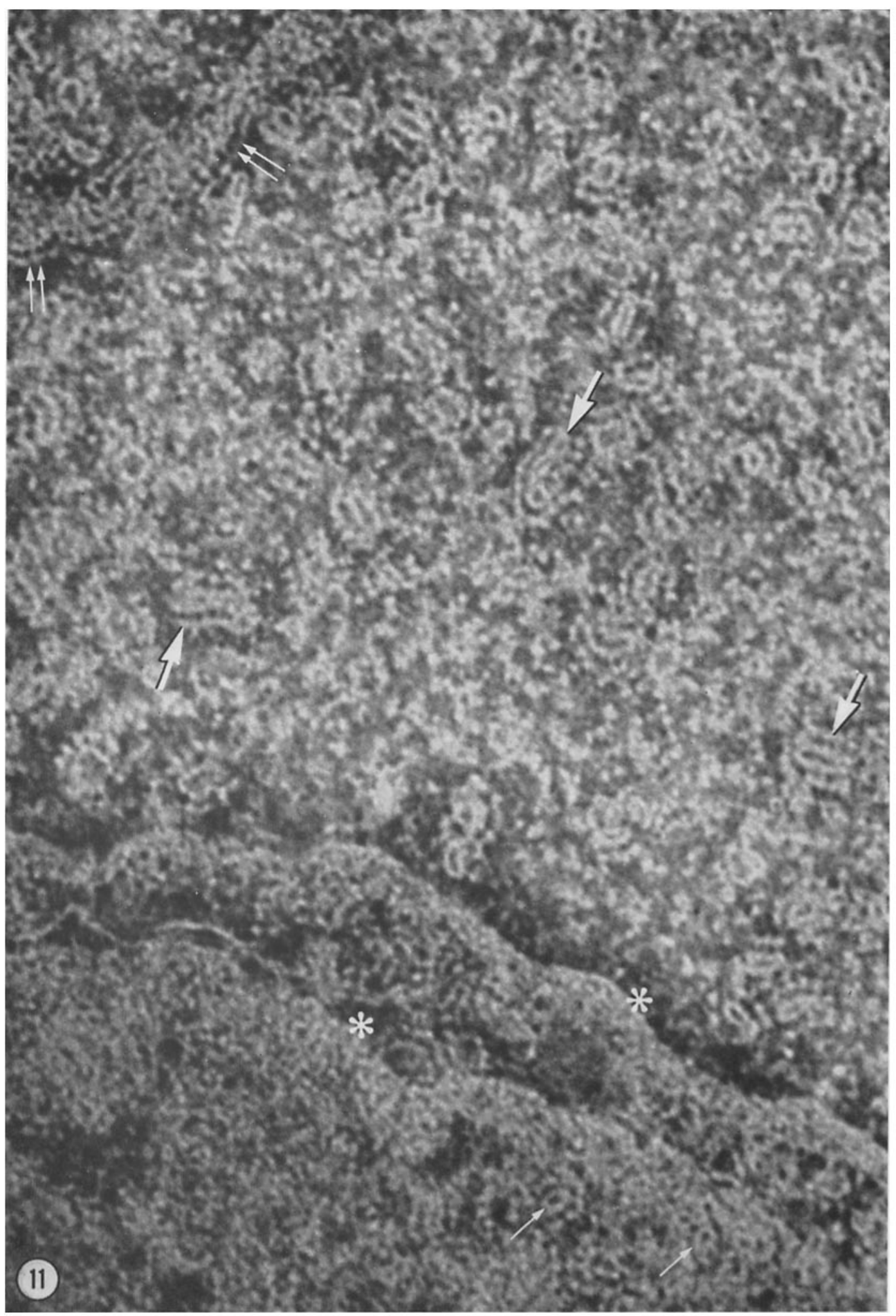

David S. Smiti Flight Muscle Sarcosomes 
"assemblies," in a configuration ensuring, within each assembly, minimal diffusion distance between the molecules of the respiratory chain. Furthermore, it has been found that, in mitochondrial fragments, the molar proportions of respiratory carriers and the amount of carrier protein per unit weight of total protein are constant, regardless of the size of the fragments (Gamble and Lehninger, 1956; Devlin and Lehninger, 1958; Green, 1959; Green and Oda, 1961; Lehninger, 1962 etc.), indicating that the respiratory assemblies are evenly dispersed throughout the membranes of the intact mitochondrion. Lehninger (1962) states that a single liver cell mitochondrion, with its sparsely distributed cristae, contains at least 10,000 assemblies, and a heart muscle sarcosome, far more.

Lehninger (1962) concludes that from 25 to 40 per cent of the membrane protein of the mitochondrion may consist of respiratory chain and coupled phosphorylating enzymes, a supposition supported by other estimates (Green and Oda, 1961; Estabrook and Holowinsky, 1961). Lehninger comments, in summary, that the "mitochondrial membranes are not simply metabolically inert 'skins,' but rather complex fabrics of regularly spaced multienzyme systems."

If the distribution of respiratory assemblies is constant, per unit area of membrane, from one type of mitochondrion to another, then the sarcosomes of insect flight muscle fibers, as judged from the close packing of their cristae, may be expected to contain an unusually large concentration of these respiratory units. This appears to be substantiated by the observed high oxygen intake of the flying insect, but it should be remembered, in this connection, that the sarcosome content of flight muscle is often very high-ca. 40 per cent of the fiber volume in the dragonfy Aeshna and the beetle Tenebrio (Smith, $1961 a, b$ ), and in
Calliphora. A more rigorous demonstration of the high concentration of respiratory assemblies in flight muscle sarcosomes is suggested by the high rate of oxygen consumption and oxidative phosphorylation of isolated sarcosomes, when supplied with the correct substrates after careful preparation of the cell fraction (cf. Van den Bergh, 1962). Although it is tempting to suppose that the particles seen in phosphotungstate preparations of Calliphora mitochondria, from their uniform size and form and characteristic distribution, represent an integral part of the structure of the intact membranes, the question of their relationship, if any, with the respiratory assemblies cannot be settled on the basis of the available evidence. It may be said, however, that while it seems possible that groups of particles might represent individual assemblies, it is uncertain whether the protein molecules constituting these units could be accommodated in single particles of the size of those described here. ${ }^{4}$

${ }^{4}$ Lehninger and coworkers (1958) suggest that the molecular weight of a respiratory assembly, assumed to consist of six carrier protein molecules and three additional enzyme protein molecules at each of the three phosphorylating sites, may be of the order of 1.5 million. Frey-Wyssling (1962) states that a particle of globular protein with a molecular weight of 144,000 (taking the density of protein as 1.3) will be $c a .70 \mathrm{~A}$ in diameter. On this basis, the diameter of a single particle, of molecular weight 1.5 million, will be ca. $165 \mathrm{~A}$. If it is assumed that the stalked particles seen in Calliphora preparations consist primarily of protein, and that the density is the same as that employed above, then the molecular weight of the $c a$. 95 A diameter "head" plus the "stalk" would not exceed 400,000.

Green and Oda (1961) have considered in detail the possible enzymatic and structural organization of the mitochondrial membranes. These investiga-

Figures 12 and 12 a A negatively stained expanse of membrane from a disrupted Calliphora sarcosome, believed to represent a portion of a crista, in surface view. The rectangular area at upper right corresponds to the area above the dotted line in Fig. $12 a$, and it is seen that beyond this line (in Fig. 12 a) the compact membrane crista structure breaks down $\left({ }^{*}\right)$ into a series of ribbons ( $c f$. Figs. 9, 10, 13, and 14). In Fig. 12, the membrane surface is interrupted by circular fenestrations or perforations (white asterisks) having sharply delimited borders. Elsewhere the membrane bears large numbers of particles, both randomly distributed and also (thick arrows) associated with circular or elongate figures ( $c f$. Fig. 11). At the points indicated with thin arrows, particle-bearing figures project into the perforations of the membrane surface; it is suggested that this circumstance may account for the indistinct structures noted within the crista fenestrations in sectioned material ( $c f$. Fig. 7). Fig. 12, $\times 120,000$; Fig. $12 a, \times 70,000$. 


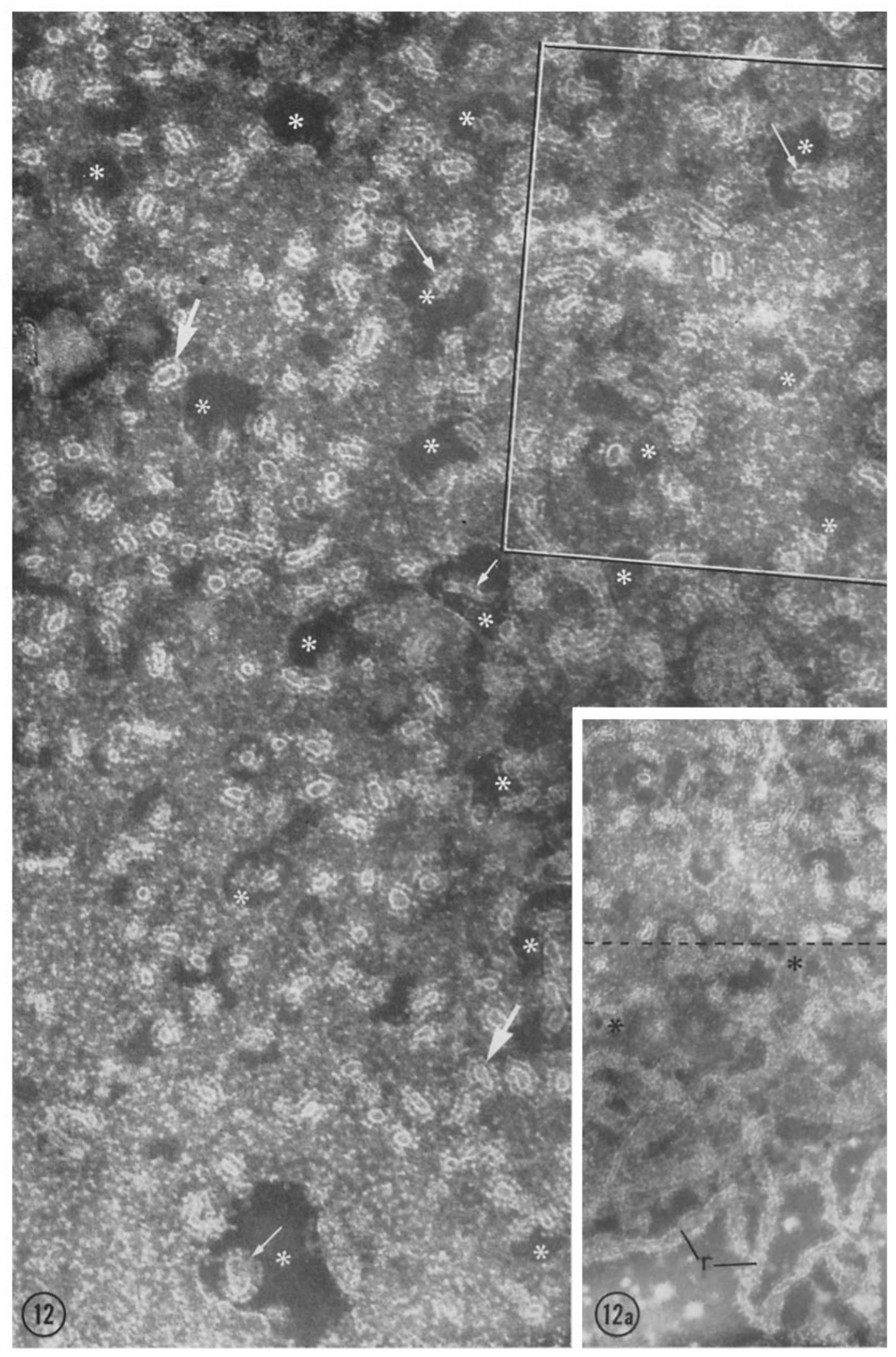

David S. SмIтH Flight Muscle Sarcosomes 
If the phosphotungstate preparations indeed reveal a genuine macromolecular differentiation of the sarcosome membranes, then it is clear that this organization either is interfered with or

tors isolated an electron transport particle (ETP) fraction from heart muscle sarcosomes. This fraction was found, in an electron microscopic study made by Fernández-Morán, to contain rectangular units representing circular or flattened cylinders, ca. $450 \mathrm{~A}$ long and $c a .100$ to $150 \mathrm{~A}$ in diameter. It was suggested that this ETP contains structural protein and lipid, in addition to the protein molecules of the electron transfer system. The molecular weight of this particle was calculated (from electron microscopic dimensions) as 3 to 6 million, and estimates of the same order of magnitude were obtained from calculations based on the determined composition of the particles, and from sedimentation data. It was further suggested that these units, comprising both active and structural moieties, may be stacked in rows in the intact mitochondrial membrane, possibly with the protein component representing the osmiophilic cristae, and the lipid component occupying the electron-transparent intracrista space.

Recently, further investigations on the constitution of mitochondrial particles have been published obscured, in conventionally fixed and sectioned material, a problem which may be overcome, in part at least, by special procedures (FernándezMorán, $1962 b, c)$. In particular, the stalked par-

by Fernández-Morán $(1962 a, b ; 1963)$ and Green et al. (1963). These authors describe the isolation from beef heart mitochondria of a homogeneous fraction consisting of structures smaller than the ETP units, in which the activities of various components of the electron transfer system are concentrated about three-fold relative to the intact mitochondrion. It was suggested that these isolated elementary particles (EP) correspond to the structures seen by negative staining procedures. FernándezMorán (1963) and Green $e t$ al. (1963) state that the molecular weight of this isolated EP is ca. $1.4 \times 10^{6}$ and that (Fernández-Morán, $1962 b$ ) the "ETP is considered to be a composite of the smaller particles invested with a coat of structural protein." However, it does not yet appear to be possible to correlate these results fully, or positively to identify the structures visualized in phosphotungstate preparations, since the diameter of the isolated particles was found to be 140 to $160 \mathrm{~A}$; considerably larger, that is, than those observed in negatively stained mitochondrial fragments from vertebrate or insect sources.

Figure 13 A portion of a ribbon from a disrupted sarcosome, negatively stained with potassium phosphotungstate. The electron-opaque stain reveals a series of spherical particles, associated with the axis of the ribbon ( $f f$. Fig. 9). The laterally placed particles are most clearly seen, but these are also present over the more expanded regions of the structure ( $c$. Fig. 15). As is more clearly seen in Fig. 14 (representing the boxed area, at higher magnification), the particles appear to be attached by stalks to the axial structure of the ribbon. $\times 310,000$.

Figure 14 A portion of the field shown in Fig. 13, at higher magnification. The particles associated with the axis of the ribbon are spherical or slightly ovoid, 80 to $95 \mathrm{~A}$ in diameter, and the negative stain clearly indicates that (as in the regions indicated by arrows) each particle is produced into a stalk, 40 to $50 \mathrm{~A}$ in length and 30 to $40 \mathrm{~A}$ in diameter, apparently linking the "head" of the particle to the axial structure. There is some indication $\left({ }^{*}\right)$ that the axis of the ribbon is differentiated, at the region of attachment of each "stalk", into a "dumbbell" configuration. Similar stalked particles also are seen in association with more or less intact cristae (from which the ribbons are thought to be derived), and on the fragments believed to represent the outer membrane of the sarcosome (Fig. 11). It seems likely that all the particles visualized in these negatively stained preparations are associated with a substrate membrane in this manner. $\times 730,000$.

Figure 15 A low-power field of a membranous ribbon from a negatively stained sarcosome preparation, isolated in deionised water, from flight muscle of the butterfly $\mathrm{Nym}$ phalis io.

In addition to the sarcosomes of Calliphora and the species illustrated here, sarcosomes from the beetle Tenebrio were also examined, and in each instance the appearance of the sarcosome fragments, after suspension in potassium phosphotungstate solution, was found to be similar. In this figure, particles flanking the ribbon axis are seen (arrows), and particles are also distributed over the more expanded portions of the ribbon axis $(*) . \times$ 110,000 


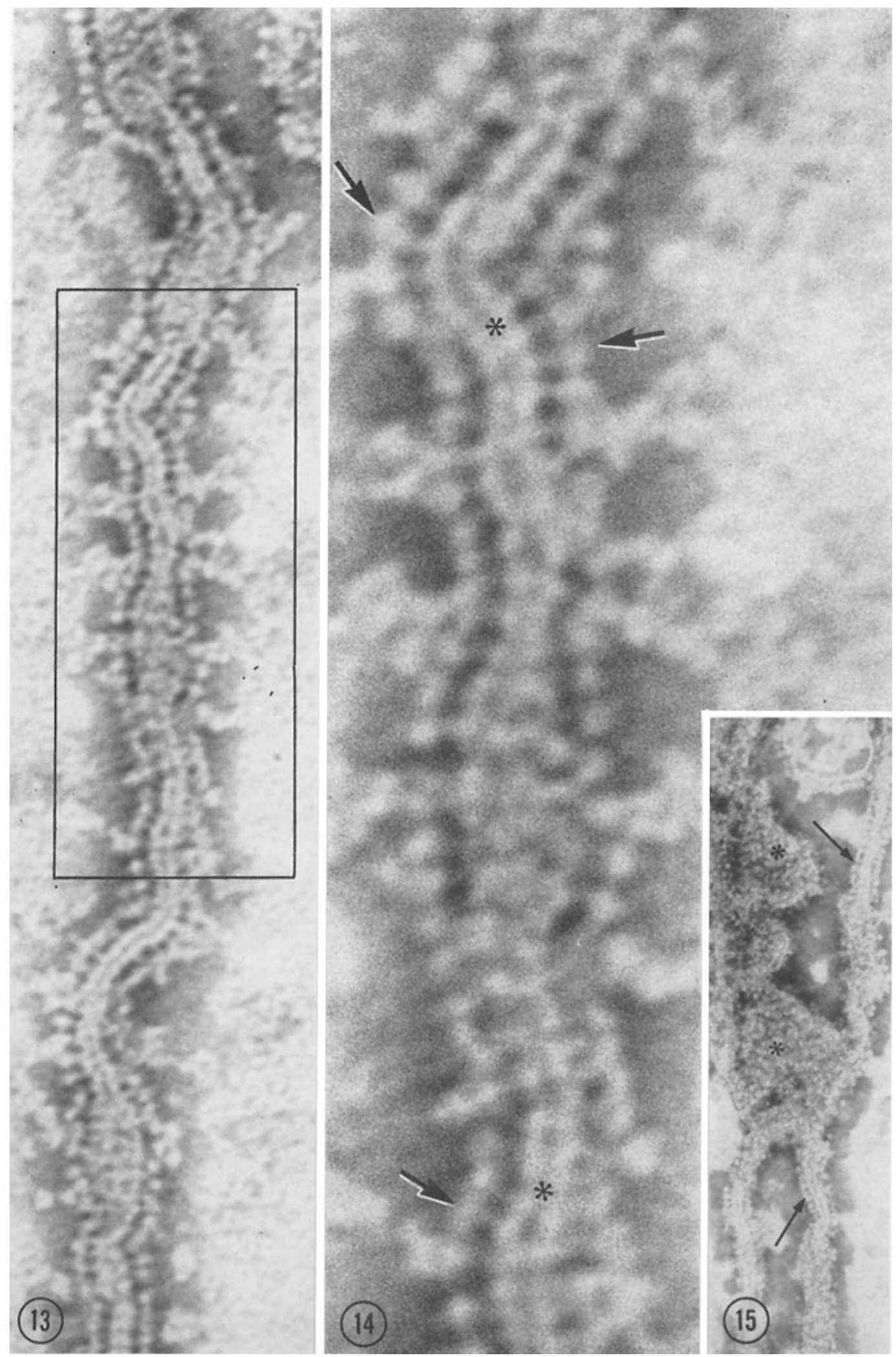




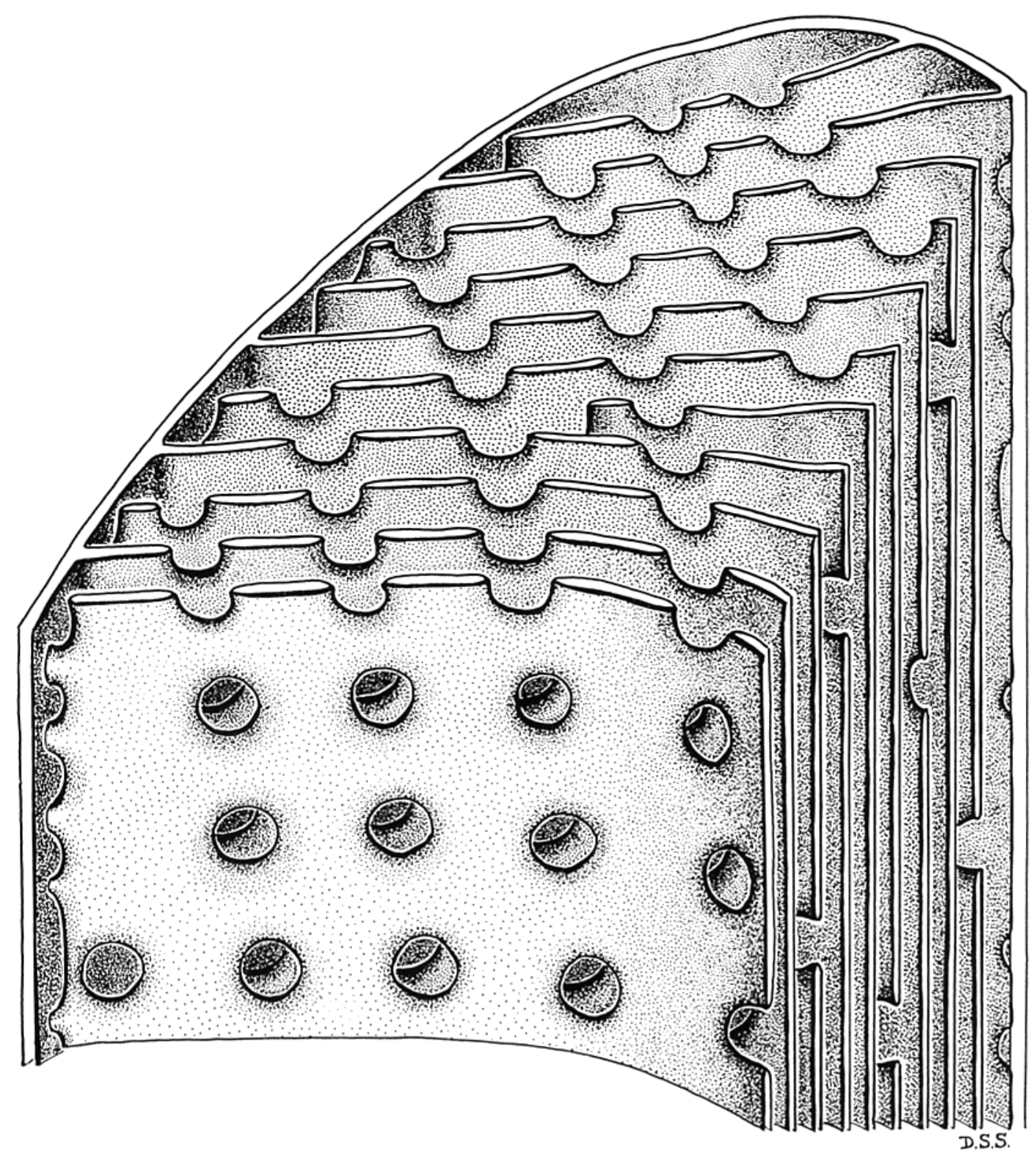

Figure 16 A semi-diagrammatic reconstruction of the three-dimensional organization of a flight muscle sarcosome of the blowfly Calliphora, based on electron micrographs of sectioned material. This drawing may be compared with Figs. 1 through 7 , and it should be noted that no attempt has been made to incorporate into this figure the evidence on membrane structure, revealed by negative staining of fragmented mitochondria.

The subparallel cristae are fenestrated, and the fenestrations are aligned to define cylindrical channels within the sarcosome matrix: these channels lie within the plane of section in the upper portion of this "block." As is indicated here, the cristae are linked, at intervals, with the inner limiting mitochondrial membrane, while the outer membrane follows an uninterrupted course around the structure.

N.B. For simplification in the diagram the space between successive cristae and the regularity of the fenestrations have been exaggerated: the actual appearance of these features may be seen in the accompanying electron micrographs. 
ticles are not seen in sectioned Calliphora sarcosomes: if they represent a structural component of the intact mitochondrion, then this organization is apparently lost during specimen preparation. At what point this occurs is not clear: the particles are present in negatively stained sarcosome fragments fixed for 30 minutes in osmium tetroxide prior to addition of the phosphotungstate, and it may be that these structures are lost during dehydration and/or embedding. Furthermore, it is by no means clear, from measurements of the components of negatively stained and sectioned material, how the particles may be integrated into the structure of the intact sarcosome. The intercrista "matrix" of the Calliphora sarcosome is ca. 70 to $100 \mathrm{~A}$ wide, and the "membranes" seen in sections of the cristae are $c a .70 \mathrm{~A}$ in thickness. The particles, with their stalks, total ca. 120 to $145 \mathrm{~A}$ in length. It is thus clear that, unless considerable shrinkage of the sarcosome phases occurs during preparation of the sections, the particles cannot simply protrude into the matrix (the electron-transparent "gap" between the cristae) from attachment sites on a $70 \mathrm{~A}$ lipoprotein membrane. The dimensions of the components derived from these two preparative techniques could perhaps be reconciled if, for example, the "matrix" seen in sectioned profiles originally contained or accommodated interdigitating series of spherical "heads" of particles associated with adjacent cristae, and if the "stalks" were to be incorporated into the osmiophilic "membranes."

\section{REFERENCES}

Andersson-Cedergren, E., J. Ultrastruct. Research, 1959, suppl. 1, 1.

Brenner, S., and Horne, R. W., Biochim. et Biophysica Acta, 1959, 34, 103.

Chance, B., and Williams, G. R., Advan. Enzymol., $1956,17,65$.

Chance, B. and Sacktor, B., Arch. Biochem. and Biophysics, 1958, 76, 509.

Devlin, T. M., and Lehninger, A. L., J. Biol. Chem., 1958, 233, 1586.

ESTABrook, R. W., and Holowinsky, A., J. Biophysic. and Biochem. Cytol., 1961, 9, 19.

FawcetT, D. W., and Revel, J. P., J. Biophysic. and Biochem. Cytol., 1961, 10, No. 4 suppl., 89.

Fernández-Morán, H., in Symposia of the International Society for Cell Biology, The Interpretation of Ultrastructure, New York, Academic Press, Inc., $1962 a, 1,411$.
At all events, it appears both from edge-on views of the ribbons derived from the cristae, and from surface aspects of the cristae and outer membrane fragments, that the particles are in some way associated with a continuous supporting structure, presumably a lipoprotein membrane.

Despite the present uncertainty attending the interpretation of these observations, it is clear that correlated studies on negatively stained and sectioned material may reveal new aspects of the organization of the mitochondrial fabric. To this end, the sarcosomes of insect flight muscle fibers, and notably those of Calliphora, afford promising material for future investigation of the physical and biochemical architecture of these organelles.

Note added in proof: Since this paper went to press, Dr. D. F. Parsons and Dr. W. Stoeckenius have described the structure of negatively stained mitochondria, respectively in Science, 1963, 140, 985 and J. Cell Biol., 1963, 17, 443. Lysed mitochondria from a variety of tissues were examined in these studies, and in every case stalked particles similar to those described above were observed, in association with the crista membranes. However, in contrast to the findings on Calliphora sarcosomes, these authors found such particles to be absent from the outer mitochondrial membranes.

This work was carried out during the author's tenure of a Fellowship from the Directory of Scientific and Industrial Research.

Received for publication, February 14, 1963.

Fernández-Morán, H., Circulation, $1962 b$, 26, 1039.

Fernández-Morán, H., Res. Publ. Ass. Nerv. Ment. Dis., $1962 c, 40,235$.

Fernández-Morán, H., Science, 1963, 140, 381, (abstr).

FreY-WyssLING, A., in Symposia of the International Society for Cell Biology, The Interpretation of Ultrastructure, New York, Academic Press, Inc., 1962, 1, 67.

Gamble, J. L., and Lehninger, A. L., J. Biol. Chem., 1956, 223, 921.

Gilmour, D., The Biochemistry of Insects, New York, Academic Press, Inc., 1961, chapt. 4.

Green, D. E., Advan. Enzymol., 1959, 21, 73.

Green, D. E., and Hatefi, Y., Science, 1961, 133, 13.

Green, D. E., and OdA, A., J. Biochem. (Tokyo), $1961,49,742$.

David S. Sмгтн Flight Muscle Sarcosomes 
Green, D. E., Blair, P. V., and Oda, T., Science, 1963, 140, 382, (abstr.).

Harman, J. W., Exp. Cell Research, 1950, 1, 394

Horne, R. W., in Techniques for Electron Microscopy, (D. D. Kay, editor), Oxford, Blackwell's Scientific Publications, 1961.

Horne, R. W. and WhitTaker, V. P., Z. Zellforsch., 1962, $\mathbf{5 8}, 1$.

Huxley, H. E. and Hanson, J., in Structure and Function of Muscle, (G. H. Bourne, editor), New York, Academic Press, Inc., 1960, 1, 183.

Köll.ıker, A., Z. Wiss. Zool., Abt. A, 1888, 47, 689.

Lehninger, A. L., Physiol. Rev., 1962, 42, 467.

Lehninger, A. L., Wadkins, C. L., Cooper, C., Devlin, T. M. and Gamble, J. L., Science, 1958, $128,450$.

Millonig, G., J. Appl. Physics 1961 a, 32, 1637.

Millonig, G., J. Biophysic. and Biochem. Cytol., 1961 $b, 11,736$.

Ogston, A. G. and Smithies, O., Physiol. Rev., 1948, 28, 283.
Porter, K. R., J. Biophysic. and Biochem. Cytol., 1961, 10, No. 4 suppl., 219.

Porter, K. R. and Palade, G. E., J. Biophysic. and Biochem. Cytol., 1957, 3, 269.

Revel, J. P., J. Biophysic. and Biochem. Cytol., 1962, 12,571 .

SAGKTor, B., J. Biophysic. and Biochem. Cytol., 1955, $1,29$.

Sмiтн, D. S., J. Biophysic. and Biochem. Cytol., 1961 a, 10, suppl., 123.

Sмiтh, D. S., J. Biophysic. and Biochem. Cytol., 1961 b, $11,117$.

Sмiтн, D. S., Rev. Canad. Biol., 1962, 21, 279.

Tiegs, O. W., Phil. Trans. Roy. Soc. London, 1955, 238, 221.

VAN den Bergh, S. G., Respiration and Energy Production in the Flight Muscle of the Housefly Musca domestica L., Doctoral thesis in the University of Amsterdam, Amsterdam, Holland, 1962.

Watanabe, M. I. and Williams, C. M., J. Gen. Physiol., 1951, 34, 675. 